\title{
Studies on nutritional, quality and sensory evaluation of value added baked products with button mushroom (Agaricus bisporus) powder
}

\author{
Monika Thakur* and Karuna Singh \\ Amity Institute of Food Technology, Amity University, NOIDA(U.P.) INDIA \\ (Email : mthakur1@ amity.edu)
}

\begin{abstract}
Mushroom has been relished as a source of food and medicine throughout the world. The fresh mushroom and dried mushroom powder was analyzed for their nutritional composition. The present study was undertaken with the objective of value addition of mushroom powder in baked products. Mushroom powder has been incorporated in the formulations of cake at $0,5,10$, 15 and 20 per cent by weight. The effect of various levels of mushroom powder on the physico-chemical properties and nutritional quality of cakes has been observed. The cakes containing mushroom powder in different concentrations were evaluated for their sensory attributes by a panel at 9 pointer hedonic scale. The results revealed that the colour and flavour of cake containing mushroom powder was significantly different from the control cake. Mushroom powder at 15 per cent addition level significantly improved colour, flavour and texture of cake. The texture and overall acceptability of the cakes with mushroom powder was equally acceptable to the control cake.
\end{abstract}

Key Words : Agaricus bisporus, Mushroom powder, Value added products, Sensory evaluation

View Point Article : Thakur, Monika and Singh, Karuna (2018). Studies on nutritional, quality and sensory evaluation of value added baked products with button mushroom (Agaricus bisporus) powder. Internat. J. agric. Sci., 14 (1) : 173-179, DOI:10.15740/HAS/IJAS/ 14.1/173-179.

Article History : Received : 30.05.2017; Revised : 27.11.2017; Accepted : 10.12.2017

* Author for correspondence: 